\title{
No Influence of Nonivamide-nicoboxil on the Peak Power Output in Competitive Sportsmen
}

\section{(ㄷ)(ㄱ) 우 $\ominus$}

\author{
Authors \\ Theresa Schörkmaier $^{1}{ }^{\mathbb{D}}$, Yvonne Wahl' ${ }^{5}$, Christian Brinkmann ${ }^{3}$, Wilhelm Bloch ${ }^{2,4}$, Patrick Wahl', 4, 6
}

\section{Affiliations}

1 Department of Clinical Research, German Centre for Neurodegenerative Diseases, Bonn, Germany

2 The German Research Centre of Elite Sport, German Sport University Cologne, Cologne, Germany

3 Department of Preventive and Rehabilitative Sport Medicine, Institute of Cardiovascular Research and Sport Medicine, German Sport University Cologne, Cologne, Germany

4 Department of Molecular and Cellular Sport Medicine, Institute of Cardiology and Sports Medicine, German Sport University Cologne, Cologne, Germany

5 Insitute of Exercise Training and Sport Informatics, German Sport University Cologne, Cologne, Germany

6 Institute of Interdisciplinary Exercise Science and Sports Medicine, Medical School Hamburg, Germany

\section{Key words}

muscle oxygenation, near-infrared spectroscopy (NIRS),

cycling, hypoxia

accepted 16.02.2021

published online 15.04 .2021

Bibliography

Int J Sports Med 2021; 42: 1092-1097

DOI $10.1055 / a-1403-2701$

ISSN $0172-4622$

(C) 2021. The Author(s).

This is an open access article published by Thieme under the terms of the Creative Commons Attribution-NonDerivative-NonCommercial-License, permitting copying and reproduction so long as the original work is given appropriate credit. Contents may not be used for commercial purposes, or adapted, remixed, transformed or built upon. (https://creativecommons. org/licenses/by-nc-nd/4.0/)

Georg Thieme Verlag KG, Rüdigerstraße 14, 70469 Stuttgart, Germany

\author{
Correspondence \\ Patrick Wahl \\ Institute of Cardiology and Sports Medicine \\ German Sport University Cologne \\ Am Sportpark Muengersdorf 6 \\ 50933 Cologne \\ Germany \\ Tel. : 00492214982 6116, Fax : 004922149828370 \\ Wahl@dshs-koeln.de
}

\section{ABSTRACT}

Recent studies have shown that the oxygenated hemoglobin level can be enhanced during rest through the application of nonivamide-nicoboxil cream. However, the effect of nonivamide-nicoboxil cream on oxygenation and endurance performance under hypoxic conditions is unknown. Therefore, the purpose of this study was to investigate the effects of nonivamide-nicoboxil cream on local muscle oxygenation and endurance performance under normoxic and hypoxic conditions. In a cross-over design, 13 athletes (experienced cyclists or triathletes [age: $25.2 \pm 3.5$ years; $\mathrm{VO}_{2} \max 62.1 \pm 7.3 \mathrm{~mL} \cdot \mathrm{min}^{-1} \cdot \mathrm{kg}^{-1}$ ]) performed four incremental exercise tests on the cycle ergometer under normoxic or hypoxic conditions, either with nonivamide-nicoboxil or placebo cream. Muscle oxygenation was recorded with near-infrared spectroscopy. Capillary blood samples were taken after each step, and spirometric data were recorded continuously. The application of nonivamide-nicoboxil cream increased muscle oxygenation at rest and during different submaximal workloads as well as during physical exhaustion, irrespective of normoxic or hypoxic conditions. Overall, there were no significant effects of nonivamide-nicoboxil on peak power output, maximal oxygen uptake or lactate concentrations. Muscle oxygenation is significantly higher with the application of nonivamide-nicoboxil cream. However, its application does not increase endurance performance. 


\begin{tabular}{|c|c|}
\hline ANOVA & Analysis of variance \\
\hline $\mathrm{F}$ & Nonivamide-nicoboxil cream \\
\hline $\mathrm{Hb}$ & Hemoglobin \\
\hline $\mathrm{HF}$ & Hypoxia-Finalgon \\
\hline HR & Heart rate \\
\hline HP & Hypoxia-Placebo \\
\hline LT & Lactate threshold \\
\hline LT1 & Lactate threshold 1 (first rise) \\
\hline LT2 & Lactate threshold $2\left(\mathrm{D}_{\max }\right)$ \\
\hline MPO & Mean power output \\
\hline NF & Normoxia-Finalgon \\
\hline NIRS & Near-infrared spectroscopy \\
\hline NP & Normoxia-Placebo \\
\hline $\mathrm{O}_{2} \mathrm{Hb}$ & Oxygenated hemoglobin \\
\hline$P$ & Placebo cream \\
\hline PPO & Peak power output \\
\hline RER & Respiratory exchange ratio \\
\hline RPE & Rating of perceived exertion \\
\hline rel. $\mathrm{VO}_{2}$ & Relative oxygen uptake \\
\hline $\mathrm{smO}_{2}$ & Saturated muscle oxygenation \\
\hline $\mathrm{SO}_{2}$ & Arterial oxygen saturation \\
\hline $\mathrm{VO}_{2 \max }$ & Maximal oxygen uptake \\
\hline W & Watt \\
\hline
\end{tabular}

\section{Introduction}

During exercise, an enormous increase in blood flow is necessary and $\mathrm{O}_{2}$ is increasingly extracted from oxygenated hemoglobin $\left(\mathrm{O}_{2} \mathrm{Hb}\right)$ to meet the oxygen $\left(\mathrm{O}_{2}\right)$ demands of the muscle cells $[1,2]$. In different conditions, e. g. hypoxia, $\mathrm{O}_{2}$ supply is a limiting factor of physical performance. With an increase in altitude and a decrease in barometric pressure, a reduction in arterial oxygen partial pressure $\left(\mathrm{pO}_{2}\right)$ occurs and thus a drop in $\mathrm{O}_{2}$ binding to hemoglobin $(\mathrm{Hb})$ [3]. The decrease in $\mathrm{O}_{2} \mathrm{Hb}$ leads to a reduced $\mathrm{O}_{2}$ supply to the muscle cell and, therefore, to reduced performance [4].

It has been shown that the systemic oxygen delivery to the locomotor muscles $[5,6]$ and the utilization of $\mathrm{O}_{2}$ in the muscle, depending on capillarization, mitochondrial density, and myoglobin content $[7,8]$, have a significant influence on endurance peak performance and maximum oxygen uptake $\left(\mathrm{VO}_{2 \max }\right)$. However, recent studies also showed that an increased local muscle blood flow, caused by beetroot juice supplementation, can improve performance [9].

The use of a blood circulation-promoting cream (nonivamidenicoboxil cream: F) could be another way to increase the $\mathrm{O}_{2}$ supply in the muscles and therefore athletes' performance. As described by Warnecke et al. [10] nonivamide (N-Vanillyl-Nonamid) is a synthetic agent related to capsaicin, which leads in combination with esters of nicotinic acid (nicoboxil) to hyperemia after application. The use of a nonivamide-nicoboxil cream increases the $\mathrm{O}_{2} \mathrm{Hb}$ level with a simultaneous (but lower) reduction of the deoxygenated hemoglobin $(\mathrm{HHb})$, and thus results in an increase of the total hemoglobin $\left(\mathrm{tHb}=\mathrm{HHb}+\mathrm{O}_{2} \mathrm{Hb}\right)$ in the muscle [10]. Earlier radioactive tracer experiments revealed an increase in muscle blood flow through the application of a nonivamide-nicoboxil [11].
However, a lack of research regarding the effects of local blood flow and oxygenation during exercise under hypoxic conditions still exists. Only a few studies have investigated the influence of increased muscular perfusion on $\mathrm{O}_{2}$ saturation in normoxia [10-12] or focused on the effects of local blood flow and muscle oxygenation on physical performance $[12,13]$. Thus, the question arises, whether the vasodilatory effect of $F$ may favor the local muscle oxygenation and increases the performance during normoxic $(N)$ and hypoxic $(\mathrm{H})$ conditions.

Therefore, this study aimed to investigate the effects of an increased local muscle oxygenation on the performance of sportsmen in hypoxia compared to normoxia using $\mathrm{F}$. In addition, we examined the change in systemic and local oxygenation and the $\mathrm{O}_{2}$ extraction of the muscles under these conditions.

\section{Materials and Methods}

\section{Participants}

13 male cyclists/triathletes [age: $25.2 \pm 3.5$ years; height: $180.4 \pm$ $5.2 \mathrm{~cm}$; mass: $71.0 \pm 8.0 \mathrm{~kg} ; \mathrm{VO}_{2 \max }: 62.1 \pm 7.3 \mathrm{~mL} \mathrm{~min}^{-1} \cdot \mathrm{kg}^{-1}$; body fat: $9.3 \pm 3.2 \%$; skinfold thickness: $5.2 \pm 1.1 \mathrm{~mm}$; (mean \pm SD)] who were experienced with laboratory testing procedures participated in this blinded, randomized, cross over study. The procedures were approved by the local ethics committee and were conducted according to international standards [14]. Each participant was informed about the procedure and protocols, and signed a declaration of agreement. Prior to all testing, the cyclists were not allowed to perform strenuous exercise, and were instructed to refrain from caffeine prior to exercise testing.

\section{Experimental Design}

To test the influence of nonivamide-nicoboxil cream (Finalgon cream, Boehringer Ingelheim GmbH \& Co. KG, Germany, containing $0.17 \%$ nonivamide and $1.08 \%$ nicoboxil) on local muscle oxygenation, blood lactate concentration, $\mathrm{O}_{2}$ uptake, and peak power output in normobaric hypoxia $\left(2800 \mathrm{~m}, 14.8 \% \mathrm{~F}_{\mathrm{i}} \mathrm{O}_{2}\right)$ and normoxia (64 $\mathrm{m}$ above sea level, $20.9 \% \mathrm{~F}_{\mathrm{i}} \mathrm{O}_{2}$ ), athletes performed four maximal graded exercise tests, within a time frame of 3 weeks and at least four days of recovery in between, on the cycle ergometer either with $\mathrm{F}$ or a placebo (Ultra-Sensitive Body Lotion, Alverde Naturkosmetik, dmdrogerie markt GmbH und Co KG, Germany; P) applied on the M. vastus lateralis of both legs.

To ensure an accurate measurement of muscle oxygenation using NIRS, only subjects with a skin thickness $<12 \mathrm{~mm}$ were allowed to participate in the study. Athletes' subcutaneous fat thickness at the vastus lateralis was identified using ultrasound (Xario XG, Toshiba, Tokyo, Japan) one week before the first exercise test. Additionally, both creams were applied on the skin to test allergic reactions.

\section{Incremental Step Test}

All participants performed four maximal graded exercise tests on cycling ergometer (Schoberer Rad Meßtechnik SRM GmbH, Jülich, Germany) in a randomized order, blinded for $\mathrm{H} / \mathrm{N}$ and $\mathrm{F} / \mathrm{P}$, consisting of cycling at a cadence $\geq 80$ revolutions per minute (rpm) with 
an initial workload of 100 watts (W) and $20 \mathrm{~W}$ increments every 3 $\min (\min )$ until volitional exhaustion. The test was terminated when the cadence decreased below $70 \mathrm{rpm}$.

Prior to the application of the creams, an area of $18 \times 12 \mathrm{~cm}$ between patella medialis and the front upper iliac spine was determined and labelled. We wanted to limit the discomfort caused by nonivamide nicoboxil (strong burning sensation on the skin) by limiting the application area. Both creams (F or P) were applied at this determined area on both thighs and covered with a wrapping film. After a 7-min rest period, creams were applied. After a 5-min exposure period, NIRS measurements began, and after an additional 5-min of rest, the incremental step test started.

The hypoxic conditions $\left(2800 \mathrm{~m}, 14.8 \% \mathrm{~F}_{\mathrm{i}} \mathrm{O}_{2}\right)$ were induced by using a normobaric hypoxic-chamber (Hypoxic Training Systems, Hypoxico, New York). The $\mathrm{O}_{2}$ and $\mathrm{CO}_{2}$ concentrations were measured during the entire period with a Dräger Multiwarn $\mathrm{O}_{2}$ and $\mathrm{CO}_{2}$ gas analyzer (Dräger, Lübeck, Germany). To keep the $\mathrm{CO}_{2}$ concentration within a physiologically tolerable range (0.03-0.3\%), a CS $2210 \mathrm{CO}_{2}$ absorber was used (SK Engineering, Kiel, Germany). To guarantee the blinding during each test, the hypoxic generators were switched on during every condition, but the $\mathrm{O}_{2}$ concentration was reduced only during the two hypoxic conditions (hypoxia with nonivamide-nicoboxil cream (HF) and hypoxia with placebo (HP)). The temperature and humidity during testing were constant at $21.3 \pm 0.1^{\circ} \mathrm{C}$ and $29.3 \pm 3.1 \%$. Independent of the testing conditions $(\mathrm{H} / \mathrm{N})$, a $15-\mathrm{min}$ acclimatization phase in the hypoxic chamber was conducted before each exercise testing.

During each test, muscle oxygenation was recorded with a nearinfrared spectroscopy (NIRS; Moxy Monitor, wave length: $680 \mathrm{~mm}$ - $800 \mathrm{~mm}$, Hutchinson, Minnesota) which was attached to the M. vastus lateralis of both legs and fixed with tape to minimize light reflection and to keep the position. Before each graded exercise test, a 2-min measurement of muscle oxygenation $\left(\mathrm{smO}_{2}\right)$ and oxygen uptake (Metalyzer 3B, Cortex Medical, Leipzig, Germany) under resting conditions was performed.

Oxygen uptake, respiratory exchange ratio (RER), and heart rate (HR) (T31, Polar Electro, Kempele, Finland) were averaged over the last 30 s of each step, and capillary blood samples for lactate analysis (EBIOplus, EKF Diagnostic Sales, Magdeburg, Germany) were taken in the last $15 \mathrm{~s}$ of each step. To determine lactate concentration, $20 \mu \mathrm{L}$ of capillary blood was directly mixed with $1 \mathrm{~mL}$ of the EBIO plus system hemolysis solution, and analyzed via an amperometric-enzymatically procedure using EBIOplus (EKF Diagnostic Sales, Magdeburg, Germany). At the same time points, the rating of perceived exertion (RPE) was assessed using the 6- to 20-point Borg scale (Borg 1970). Furthermore, arterial oxygen saturation $\left(\mathrm{SO}_{2}\right)$ was recorded continuously at the fingertip with a pulse oximeter (Philips C3 Patient Monitor, Amsterdam, Netherlands). During each test, participants did not receive any feedback about the current power output or the total duration. After volitional exhaustion, a visual analog scale was used to assess the pain in leg muscles, and subjects were asked to evaluate the testing condition ( $\mathrm{H}$ or $\mathrm{N}$ ).

To compare the different testing conditions, peak power output during NP $\left(\mathrm{PPO}_{\mathrm{NP}}\right)$ was set at $100 \%$. Afterwards, parameters were compared at rest $(R)$, at $50 \%$, and $75 \%$ of the $P P O_{N P}$.
Values at physical exhaustion (100\%) during each condition were also compared irrespective of different workloads. Lactate thresholds have been determined using the method of Bishop et al. [15] ( $\left.D_{\max }\right)$.

\section{Statistical analysis}

Data were tested for normality with the Kolmogorov-Smirnov test with Lilliefors-correction. To assess the differences between the different testing conditions (NP, NF, HP, HF), a two-way analysis of variance ["altitude (oxygen concentration)" ( $N, H)$; “cream” (P, F)] repeated-measures ANOVA with Bonferroni post-hoc test was used for each power output separately (R, 50\%, 75\%, 100\%). Descriptive statistics are expressed as means \pm standard deviation (SD). All statistical analyses were performed using the software Statistica (Statistica for Windows, Version 7.0., StatSoft, Tulsa, USA).

\section{Results}

- Table 1 shows the peak power output (PPO), maximal relative oxygen uptake (rel. $\left.\mathrm{VO}_{2 \max }\right)$, and maximal heart rate $\left(\mathrm{HR}_{\max }\right)$ of the different conditions. No significant differences in $\mathrm{PPO}$, rel. $\mathrm{VO}_{2 \max }$ and $\mathrm{HR}_{\max }$ were present between $\mathrm{F}$ and $\mathrm{P}$ during $\mathrm{N}$ or $\mathrm{H}$.

\section{PPO}

Over-all ANOVA (analysis of variance) revealed a significant effect of "altitude" on PPO ( $p<0.001$ ), no significant effect of "cream" on PPO, and no significant interaction effect for any of the conditions (“altitude" * “cream"). Hypoxia significantly decreased PPO.

\section{HRmax}

The overall ANOVA revealed no significant effect of "altitude" on HRmax, no significant effect of "cream" on HRmax, and no significant interaction effect for any of the conditions ("altitude" * “cream").

\section{Saturated muscle oxygenation $\left(\mathrm{smO}_{2}\right)$}

The overall ANOVA revealed a significant effect of "altitude" on $\mathrm{smO}_{2}$ under resting conditions ( $\left.\mathrm{p}<0.001\right)$, at $50 \%(\mathrm{p}<0.001)$ and $75 \%(p<0.001)$ of $\mathrm{PPO}_{\mathrm{NP}}$ and at $100 \%(\mathrm{p}<0.01)$, a significant effect of "cream" on $\mathrm{smO}_{2}$ under resting conditions ( $\left.p<0.001\right)$, at $50 \%$ $(p<0.001)$ and $75 \%(p<0.01)$ of $\mathrm{PPO}_{N P}$ and at $100 \%(p<0.01)$, but no significant interaction effect for any of the conditions ("altitude" * “cream"). $\mathrm{smO}_{2}$ was reduced by hypoxia and increased by cream at the different workloads ( $\triangleright$ Fig. 1 ).

- Table 1 Results of the incremental exercise test during different conditions.

\begin{tabular}{|c|c|c|c|}
\hline & PPO [W] & $\begin{array}{l}\mathrm{VO}_{2 \max } \\
{\left[\mathrm{mL} \cdot \mathrm{min}^{-1} \cdot \mathrm{kg}^{-1}\right]}\end{array}$ & $\begin{array}{l}H_{\text {max }} \\
{\left[\mathrm{min}^{-1}\right]}\end{array}$ \\
\hline NP & $307 \pm 35$ & $62.1 \pm 7.3$ & $181 \pm 10$ \\
\hline NF & $313 \pm 33$ & $62.0 \pm 6.3$ & $181 \pm 11$ \\
\hline HP & $274 \pm 28^{\#}$ & $52.8 \pm 4.7^{\#}$ & $180 \pm 9$ \\
\hline $\mathrm{HF}$ & $278 \pm 31^{\#}$ & $53.7 \pm 5.8^{\#}$ & $180 \pm 9$ \\
\hline
\end{tabular}

PPO: peak power output, $\mathrm{VO}_{2 \max }$ : maximal oxygen uptake, $\mathrm{HR}_{\max }$ : maximal heart rate. \# significantly different compared with normoxia when using the same cream $(p<0,05)$. Data are shown as mean $\pm S D$. 


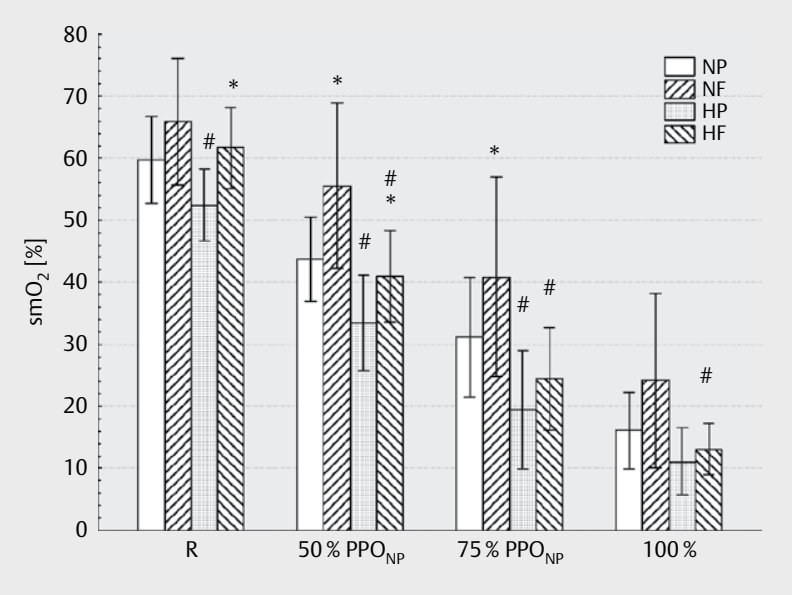

- Fig. 1 Saturated muscle oxygenation $\left(\mathrm{smO}_{2}\right)$ under resting conditions, at $50 \%$ and $75 \%$ of the peak power output at normoxia placebo $\left(\mathrm{PPO}_{\mathrm{NP}}\right)$ and after exhaustion $(100 \%)$ in each condition * significantly different between different creams at the same altitude (NP vs. NF \& HP vs. HF). \#significantly different between hypoxia and normoxia when using the same cream (NP vs. HP \& NF vs. HF) $(p<0.05)$. Data are shown as mean \pm SD. NP: Normoxia Placebo; NF: Normoxia Finalgon; HP: Hypoxia Placebo; HF: Hypoxia Finalgon.

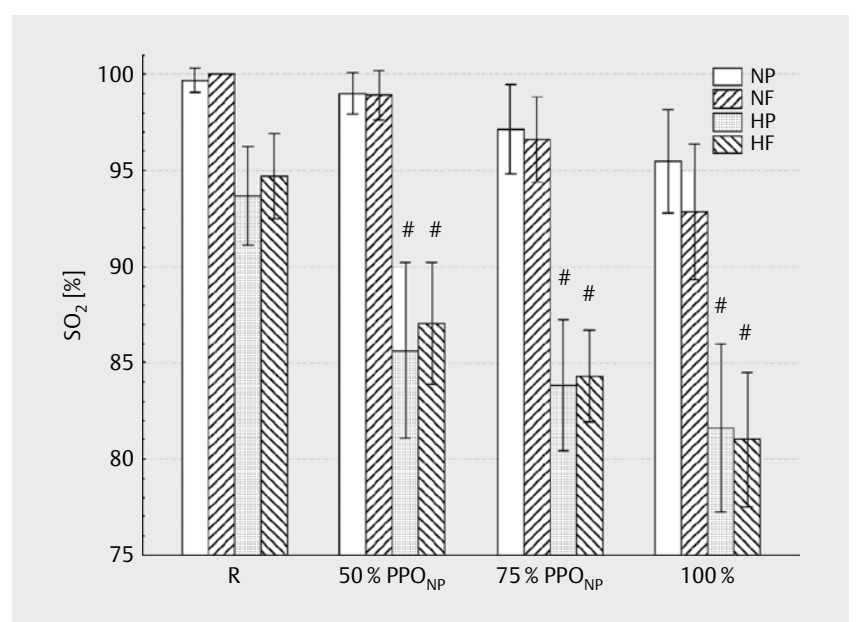

- Fig 2 Arterial oxygen saturation $\left(\mathrm{SO}_{2}\right)$ under resting conditions, at $50 \%$ and $75 \%$ of the peak power output at normoxia placebo $\left(\mathrm{PPO}_{\mathrm{NP}}\right)$ and after exhaustion (100\%) in each condition. \#significantly different between hypoxia and normoxia when using same cream (NP vs. HP \& NF vs. HF) $(\mathrm{p}<0.05)$. Data are shown as mean \pm SD. NP: Normoxia Placebo; NF: Normoxia Finalgon; HP: Hypoxia Placebo; HF: Hypoxia Finalgon.

\section{Arterial oxygen saturation $\left(\mathrm{SO}_{2}\right)$}

The overall ANOVA revealed a significant effect of "altitude" on $\mathrm{SO}_{2}$ at $50 \%(\mathrm{p}<0.0001)$, and $75 \%(\mathrm{p}<0.001)$ of $\mathrm{PPO}_{\mathrm{NP}}$ and at $100 \%$ $(p<0.001)$, a significant effect of "cream" on $\mathrm{SO}_{2}$ at $100 \%(p<0.05)$, but no significant interaction effect for any of the conditions. $\mathrm{SO}_{2}$ was reduced by hypoxia and reduced by $\mathrm{F}$ at the before mentioned workloads ( Fig. 2).

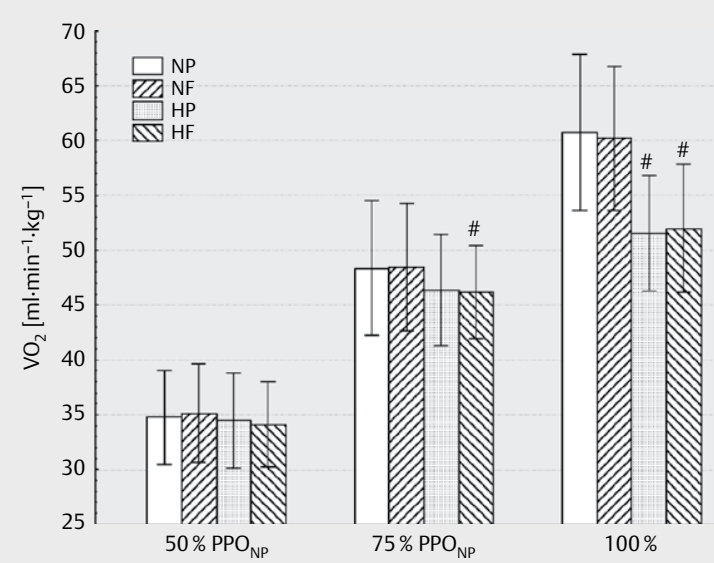

- Fig 3 Rel. Oxygen uptake $\left(\mathrm{VO}_{2}\right)$ during the different conditions at $50 \%$ and $75 \%$ of the peak power output at normoxia placebo $\left(\mathrm{PPO}_{\mathrm{NP}}\right)$ and after exhaustion (100\%) in each condition. Data are shown as mean \pm SD. \#significantly different between different altitudes when using the same cream (NP vs. HP \& NF vs. HF) $(p<0.05)$. NP: Normoxia Placebo; NF: Normoxia Finalgon; HP: Hypoxia Placebo; HF: Hypoxia Finalgon.

\section{Oxygen uptake $\left(\mathrm{VO}_{2}\right)$}

The overall ANOVA revealed a significant effect of altitude on $\mathrm{VO}_{2}$ at $75 \%$ of $\mathrm{PPO}_{\mathrm{NP}}(\mathrm{p}<0.001)$ and at $100 \%(\mathrm{p}<0.001)$, but no significant effect of "cream" on $\mathrm{VO}_{2}$ for any of the time points and no significant interaction effect for any of the conditions. $\mathrm{VO}_{2}$ was reduced by hypoxia at the before mentioned workloads ( $\vee$ Fig. 3 ).

\section{Lactate}

The overall ANOVA revealed a significant effect of altitude on lactate concentration at $50 \%(p<0.001), 75 \%(p<0.001)$ of $\mathrm{PPO}_{\mathrm{NP}}$, and at $100 \%(\mathrm{p}<0.01)$, but no significant effect of "cream" on lactate concentration at any of the time points and no significant interaction effect for any of the conditions. Hypoxia significantly increased lactate levels at different workloads ( $\bullet$ Table 2 ).

\section{Lactate Threshold (LT)}

The overall ANOVA revealed a significant effect of "altitude" on lactate threshold 1 (LT1) and lactate threshold 1 (LT2) $(\mathrm{p}<0.001)$, but no significant effect of "cream" on LT1 ( $p=0.17)$ and LT2 ( $p=0.93)$, and no significant interaction effect for any of the conditions. Hypoxia significantly decreased workload at LT1 and LT2 (

\section{Visual Analog Scale (VAS)}

The overall ANOVA revealed no significant effect of "altitude" on muscular and cardiopulmonal exertion ( $p=0.11, p=0.11)$, no significant effect of "cream" on muscular and cardiopulmonal exertion ( $p=0.15, p=0.57$ ), and no significant interaction effect for any of the conditions.

\section{Rating of Perceived Exertion (RPE)}

The overall ANOVA revealed no significant effect of "altitude" $(p=0.39)$ on RPE, no significant effect of "cream" on RPE ( $p=0.39)$, and no significant interaction effect for any of the conditions. 
- Table 2 Lactate concentration at 50 and $75 \%$ of the $\mathrm{PPO}_{\mathrm{NP}}$ and after exhaustion (100\%) and LT1 and LT2 in each condition.

\begin{tabular}{|c|c|c|c|c|c|}
\hline & $\mathbf{5 0} \% \mathbf{P P O}_{\mathbf{N P}}\left[\mathbf{m m o l} \cdot \mathbf{L}^{-1}\right]$ & $\mathbf{7 5} \% \mathbf{P P O}_{\mathbf{N P}}\left[\mathbf{m m o l} \cdot \mathbf{L}^{-1}\right]$ & $\mathbf{1 0 0} \%\left[\mathbf{m m o l} \cdot \mathbf{L}^{-1}\right]$ & $\mathbf{L T 1}(\mathbf{f i r s t} \mathbf{r i s e})[\mathbf{W}]$ & $\mathbf{L T 2}\left(\mathbf{d}_{\mathbf{m a x}}\right)[\mathbf{W}]$ \\
\hline $\mathrm{NP}$ & $1.1 \pm 0.7$ & $2.8 \pm 1.2$ & $9.8 \pm 2.0$ & $198 \pm 40$ & $259 \pm 41$ \\
\hline $\mathrm{NF}$ & $1.2 \pm 0.8$ & $2.8 \pm 1.6$ & $9.3 \pm 2.5$ & $200 \pm 43$ & $257 \pm 51$ \\
\hline $\mathrm{HP}$ & $1.8 \pm 0.9^{\#}$ & $5.9 \pm 2.1^{\#}$ & $12.0 \pm 3.2^{\#}$ & $155 \pm 30^{\#}$ & $223 \pm 28^{\#}$ \\
\hline $\mathrm{HF}$ & $1.8 \pm 0.9^{\#}$ & $5.0 \pm 2.3^{\#}$ & $11.7 \pm 1.9^{\#}$ & $165 \pm 27^{\#}$ & $227 \pm 32^{\#}$ \\
\hline
\end{tabular}

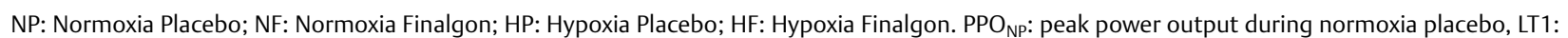
lactate threshold 1, LT2: lactate threshold 2. \# significantly different between hypoxia and normoxia when using the same cream (NP vs. HP \& NF vs. $\mathrm{HF})(\mathrm{p}<0.05)$. Data are shown as mean \pm SD.

\section{Discussion}

The present study aimed to identify the effects of the application of $\mathrm{F}$ on local muscle oxygenation and $\mathrm{O}_{2}$-extraction of the muscles, the mechanical peak power output, and the systemic oxygenation during exercise under hypoxic and normoxic conditions. The main findings of the present study are a significant increase of $\mathrm{smO}_{2}$ from the application of $\mathrm{F}$ at $50 \%$ of $\mathrm{PPO}_{\mathrm{NP}}$ in hypoxia and normoxia, and at $75 \%$ of $\mathrm{PPO}_{\mathrm{NP}}$ under normoxia compared to P. However, no significant effect on PPO, lactate concentrations, and rel. $\mathrm{VO}_{2 \max }$ has been shown, neither in hypoxia nor in normoxia. A significant difference between the effects of hypoxia and normoxia was found for almost all parameters.

In the present study, the application of $\mathrm{F}$ led to a significantly higher $(\mathrm{p}<0.01) \mathrm{smO}_{2}$ during hypoxia at rest $(\mathrm{HF})$ and submaximal intensities ( $50 \%$ and $75 \%$ of $\mathrm{PPO}_{\mathrm{NP}}$ ). As mentioned in the introduction, the physiological mechanisms induced by the application of $F$ are not yet sufficiently clarified [10]. The results of the present study are in line with the results of Warnecke et al. [10] who showed an increased $\mathrm{O}_{2} \mathrm{Hb}$ and $\mathrm{O}_{2}$-saturation in the region cruris posterior above of the M. gastrocnemius and M. soleus after the application of $F$ under resting conditions. In contrast, Zinner et al. [12] detected no increase of $\mathrm{O}_{2}$ saturation in the $\mathrm{M}$. vastus lateralis. The different results could be due to the fact that Zinner et al. [12] applied $F$ immediately after a 3-min warm-up followed by a baseline measurement. As known, physical activity leads to higher metabolic work and increased muscle blood flow [16] to cover the $\mathrm{O}_{2}$-demands [2], which may have already led to expanded resting/baseline values. In contrast to Zinner et al. [12], athletes in the present study and the study by Warnecke et al. [10] did not warm up before the rest measurement with the application of $\mathrm{F}$ to exclude possible preloads and an increased blood flow.

Concerning performance, results are in line with previous studies. Even though performance tests and exercise time differ markedly, the results are in line with the study of Zinner et al. [12], who also found no significant increase in performance in a $4 \mathrm{~km}$ time trial (TT) from the application of $F$. The results of Zinner et al. [12] showed a mean power output (MPO) of $325 \pm 59 \mathrm{~W}$ from the application of $F$, which was similar to the MPO during $\mathrm{P}(326 \pm 60 \mathrm{~W})$ and control (no cream) $(321 \pm 60 \mathrm{~W})$. However, as the application of $\mathrm{F}$ led to a significantly higher $\mathrm{smO} 2$ at submaximal intensities $(50 \%$ and $75 \%$ of $\mathrm{PPO}_{\mathrm{NP}}$ ), the question arises whether performance could be sustained longer for continuous workloads at submaximal intensities with the application of $\mathrm{F}$.

Generally, the results concerning the influence of hypoxia on performance are in line with the previous literature. Peltonen et al.
[17] indicated a significant decrease of PPO $(-12.8 \%)$ in hypoxia $(\sim 2800 \mathrm{~m}, 15.0 \% \mathrm{FiO} 2)$ compared to performance in normoxia. These findings are comparable to the present results, where the application of $\mathrm{F}$ in $\mathrm{H}$ led to a decrease of PPO of $11.2 \%$ while using the $\mathrm{P}$ led to a drop of $10.7 \%$

$\mathrm{VO}_{2 \max }$ is considered to be limited by various factors including pulmonary diffusion capacity, cardiac output, $\mathrm{O}_{2}$ transport capacity, and skeletal muscles [18]. Here, even though a higher $\mathrm{smO}_{2}$ by the topical application of $\mathrm{F}$ was measured, it did not significantly influence $\mathrm{VO}_{2 \max }$, neither in $\mathrm{N}$ nor in $\mathrm{H}$. Saltin and Calbet [5] pointed out that the $\mathrm{VO}_{2 \max }$ is limited by the systemic $\mathrm{O}_{2}$-transport into the muscles. This finding by Saltin and Calbet [5] is supported by the present study. Despite a significantly increased local muscle oxygenation and thus an increased $\mathrm{O}_{2}$-supply, there was no significant increase in $\mathrm{VO}_{2 \max }$. These findings suggest that the increased $\mathrm{O}_{2}$ availability in the muscle could not be utilized. However, it has to be mentioned, that the area where the cream was applied was perhaps too small to elicit changes in $\mathrm{VO}_{2}$ max or performance, which is a clear limitation of our study.

\section{Conclusion}

During submaximal intensities in normoxia (50\% and $75 \%$ PPONP) and hypoxia ( $50 \%$ PPONP) muscle oxygenation of the M. vastus lateralis was significantly increased through the application of $\mathrm{F}$ compared to P. However, the topical application of F prior to an incremental step test does not affect peak power output, arterial oxygen saturation or oxygen uptake of experienced cyclists, showing that the increased $\mathrm{O}_{2}$ availability in the muscle cannot be utilized.

\section{Conflict of Interest}

The authors declare that they have no conflict of interest.

References

[1] Ferrari M, Binzoni T, Quaresima V. Oxidative metabolism in muscle. Philos Trans R Soc Lond B Biol Sci 1997; 352: 677-683

[2] Sarelius I, Pohl U. Control of muscle blood flow during exercise: local factors and integrative mechanisms. Acta Physiol Scand 2010; 199: 349-365

[3] West JB, Schoene RB, Luks AM et al. High Altitude Medicine and Physiology. 5th ed.T] International Ltd; Great Britain: 2013 
[4] Richardson RS, Leigh JS, Wagner PD et al. Cellular PO2 as a determinant of maximal mitochondrial $\mathrm{O} 2$ consumption in trained human skeletal muscle. J Appl Physiol (1985) 1999; 87: 325-331

[5] Saltin B, Calbet LA. Point: In health and in a normoxic environment, VO2max is limited primarily by cardiac output and locomotor muscle blood flow. J Appl Physiol (1985) 2006; 100: 744-748

[6] Gore C], Little SC, Hahn AH et al. Reduced performance of male and female athletes at $580 \mathrm{~m}$ altitude. Eur J Appl Physiol 1997; 75: 136-143

[7] Vogt M, Flück M, Hoppeler H. "Living low - Training high": Eine effektive Höhentrainingsmethode zur Verbesserung der sportlichen Leistungsfähigkeit von trainierten Athleten. Schweiz Z Med Traumatol 2005; 53: 76-81

[8] Bhambhani Y. Muscle oxygenation trends during dynamic exercise measured by near infrared spectroscopy. Can J Appl Physiol 2004; 29: 504-523

[9] Dominguez R, Cuenca E, Maté-Muñoz JL. Effects of beetroot juice supplementation on cardiorespiratory endurance in athletes. A systematic review. Nutrients 2006; 9: 2-18

[10] Warnecke J, Wendt T, Winkler $S$ et al. Evaluation of haemoglobin changes of skin and muscle tissue of the calf induces by topical application of a nonivamide/nicoboxil cream. Can J Physiol Pharmacol 2006; 92: 149-154

[11] Hiller J, Strauss E, Jakob A. Zur Resorption markierter Salzlösungen unter der Wirkung hyperämisierender Substanzen. Untersuchungen mit J131. Arzneim Forsch 1953; 3: 515-551
[12] Zinner C, Holmberg HC, Sperlich B. Topical application of cream containing nonivamide and nicoboxil does not enhance the performance of experienced cyclists during a 4-km time-trial. Eur J Appl Physiol 2016; 116: 969-974

[13] Subudhi A, Dimmen A, Roach R. Effects of acute hypoxia on cerebral and muscle oxygenation during incremental exercise. J Appl Physiol (1985) 2007; 103: 177-183

[14] Harriss DJ, Macsween A, Atkinson G. ethical standards in sport and exercise science research: 2020 update. Int J Sports Med 2019; 40: 813-817

[15] Bishop D, Jenkins DG, Mackinnon LT. The relationship between plasma lactate parameters, W-peak and 1-h cycling performance in women. Med Sci Sports Exerc 1998; 3: 1270-1275

[16] Delph MD, Laughlin MH. Regulation of skeletal muscle perfusion during exercise. Acta Physiol Scand 1998; 162: 411-419

[17] Peltonen JE, Tikkanen HO, Rusko HK. Cardiorespiratory responses to exercise in acute hypoxia, hyperoxia and normoxia. Eur J Appl Physiol 2001; 85: 82-88

[18] Bassett D, Howley E. Limiting factors for maximum oxygen uptake and determinants of endurance performance. Med Sci Sports Exerc 1999; 32: $70-82$ 